\title{
Positivity in Multifield Effective Field Theories
}

\author{
$\mathrm{Xu} \mathrm{Li}{ }^{1,{ }^{* *}}$ Hao $\mathrm{Xu},{ }^{2,3, \dagger}$ Chengjie Yang, ${ }^{1, \$}$ Cen Zhang, ${ }^{1,4, \S}$ and Shuang-Yong Zhou $\oplus^{2,3, \|}$ \\ ${ }^{1}$ Institute for High Energy Physics and School of Physical Sciences, University of Chinese Academy of Sciences, \\ Beijing 100049, China \\ ${ }^{2}$ Interdisciplinary Center for Theoretical Study, University of Science and Technology of China, Hefei, Anhui 230026, China \\ ${ }^{3}$ Peng Huanwu Center for Fundamental Theory, Hefei, Anhui 230026, China \\ ${ }^{4}$ Center for High Energy Physics, Peking University, Beijing 100871, China
}

(Received 24 January 2021; revised 5 April 2021; accepted 24 August 2021; published 17 September 2021)

\begin{abstract}
We discuss the general method for obtaining full positivity bounds on multifield effective field theories (EFTs). While the leading order forward positivity bounds are commonly derived from the elastic scattering of two (superposed) external states, we show that, for a generic EFT containing three or more low-energy modes, this approach only gives incomplete bounds. We then identify the allowed parameter space as the dual to a spectrahedron, constructed from crossing symmetries of the amplitude, and show that finding the optimal bounds for a given number of modes is equivalent to a geometric problem: finding the extremal rays of a spectrahedron. We show how this is done analytically for simple cases and numerically formulated as semidefinite programming (SDP) problems for more complicated cases. We demonstrate this approach with a number of well-motivated examples in particle physics and cosmology, including EFTs of scalars, vectors, fermions, and gravitons. In all these cases, we find that the SDP approach leads to results that either improve the previous ones or are completely new. We also find that the SDP approach is numerically much more efficient.
\end{abstract}

DOI: 10.1103/PhysRevLett.127.121601

Introduction.-Positivity bounds are constraints on the Wilson coefficients of an effective field theory (EFT) that can be bootstrapped from fundamental properties of the $S$ matrix of the UV theory [1-3]. Recently, there has been a lot of interest in extending the strength and scope of the positivity bounds [4-19], as well as applying the bounds to constrain EFTs in various contexts (see, for example, [20-50]). In many situations, and particularly for constraining the parameter space of the standard model effective field theory (SMEFT) $[4,20-22,29,35,43]$, the leading positivity bounds for the $s^{2}$ terms ( $s, t$ being the standard Mandelstam variables) in the amplitude are phenomenologically the most relevant ones. The most widely used positivity bounds so far are based on the forward $(t=0)$ elastic scattering of two factorized states, each of which can be an arbitrary mixture of various particle modes. However, it has been shown that this approach does not always give the best bounds [4]. In addition, obtaining the complete set of superposed elastic bounds is known to be nondeterministic-polynomial hard (NP hard) [30].

In this Letter, we will establish a geometric method for obtaining the full set of leading forward positivity bounds

Published by the American Physical Society under the terms of the Creative Commons Attribution 4.0 International license. Further distribution of this work must maintain attribution to the author(s) and the published article's title, journal citation, and DOI. Funded by SCOAP. for EFTs with multiple low-energy modes. It applies not only to the SMEFT, but also to all other EFTs that involve multiple particles or multiplet particles. We will compare with the previous results and show how the new, nonelastic bounds arise from scattering entangled states.

Notations.-We will use capital calligraphy letters to denote rank-4 tensors (e.g., $\mathcal{T} \in \mathbb{R}^{n^{4}}$ ). The inner product of tensors is defined by $\mathcal{T}_{1} \cdot \mathcal{T}_{2} \equiv \sum_{i j k l} \mathcal{T}_{1}^{i j k l} \mathcal{T}_{2}^{i j k l}$. We say that $\mathcal{T}$ is positive semidefinite (PSD) if $\mathcal{T}^{i j k l}$ is a PSD matrix when $i j$ is viewed as one index and $k l$ another, which is denoted by $\mathcal{T} \succeq 0$. The null space of this matrix is denoted as null $(\mathcal{T})$. $\mathbf{S}_{+}^{n \times n}$ is the set of $n \times n$ PSD matrices. We denote by $\overrightarrow{\mathbf{S}}^{n^{4}}$ the set of rank- $4 n$-dimensional tensors $\mathcal{T}$ that satisfy the following crossing symmetries:

$$
\mathcal{T}^{i j k l}=\mathcal{T}^{i l k j}=\mathcal{T}^{k j i l}=\mathcal{T}^{j i l k} .
$$

$\mathcal{T}^{i(j|k| l)} \equiv \mathcal{T}^{i j k l}+\mathcal{T}^{i l k j}$. The set of extremal rays (ERs) of some convex cone $\mathbf{X}$ is denoted as $\operatorname{ext}(\mathbf{X})$. An ER is an element of $\mathbf{X}$ that cannot be split into two linearly independent elements inside $\mathbf{X}$.

We shall consider the $t \rightarrow 0$ limit of a two-to-two amplitude, $\mathbf{M}_{i j \rightarrow k l}(s)=\mathbf{M}_{i j \rightarrow k l}(s, t=0)$, which is only a function of $s$, and we define the $\mathcal{M}$ tensor

$$
\mathcal{M}^{i j k l} \equiv \lim _{s \rightarrow 0} \frac{d^{2}}{d s^{2}} \mathbf{M}_{i j \rightarrow k l}(s) .
$$


Here $i, j, k$, and $l$ are indices for the low-energy degrees of freedom, enumerating particle species, polarization, and other quantum numbers. We will simply call this $\mathcal{M}$ tensor "amplitude."

Dispersion relation.-Axiomatic principles of the UV amplitude, such as analyticity, unitarity, and crossing symmetry, lead to a dispersion relation that expresses $\mathcal{M}^{i j k l}$ in terms of an integral of the discontinuity of the amplitude along the positive real $s$ axis (see, e.g., [4])

$\mathcal{M}^{i j k l}=\int_{(\epsilon \Lambda)^{2}}^{\infty} \frac{d \mu \operatorname{Disc}_{i j \rightarrow k l}(\mu)}{2 i \pi \mu^{3}}+(j \leftrightarrow l)+$ c.c.

where $(j \leftrightarrow l)$ denotes the previous term with $j$ and $l$ swapped. This assumes that a self-conjugate particle basis is chosen, which is always possible by replacing $|i\rangle$ and $|\bar{i}\rangle$ by $(|i\rangle+|\bar{i}\rangle) / 2$ and $(|i\rangle-|\bar{i}\rangle) /(2 i) . \epsilon \Lambda$ is the subtraction scale for improved positivity, below which the EFT is valid: we have slightly changed the definition of $\mathcal{M}^{i j k l}$ by subtracting the dispersive integral below $\epsilon \Lambda$, see more explanations in Ref. [4]. Upon using the generalized optical theorem, this relation implies that $\mathcal{M}^{i j k l}$ is a convex cone generated from positive linear combinations of elements of the form $m^{i j} m^{k l}+m^{i l} m^{k j}$ [4], i.e.,

$$
\mathbf{C}^{n^{4}}=\operatorname{cone}\left(\left\{m^{i(j} m^{|k| l)}, m \in \mathbb{R}^{n^{2}}\right\}\right) .
$$

The elements of $\mathbf{C}^{n^{4}}$ are invariant under $(j \leftrightarrow l)$ and $\left(i \leftrightarrow k\right.$ ) exchanges. We will also assume that $m^{i j}$ is either symmetric or antisymmetric, which is simply Bose symmetry for scalars, but implies parity conservation for vectors. This is equivalent to further requiring $\mathbf{C}^{n^{4}} \subset \overrightarrow{\mathbf{S}}^{n^{4}}$.

Positivity bounds arise as the boundary of $\mathbf{C}^{n^{4}}$. All components of $\mathcal{M}$ can be computed in terms of Wilson coefficients, so bounds on $\mathcal{M}$ can be converted to bounds on these coefficients. Conventionally, these bounds are derived by the elastic scattering of a pair of factorized but arbitrarily superposed states, $|u\rangle=\sum_{i} u_{i}|i\rangle$ and $|v\rangle=\sum_{i} v_{i}|i\rangle$ : $u^{i} v^{j} u^{k} v^{l} \mathcal{M}^{i j k l} \geq 0, \quad$ thanks to $u^{i} v^{j} u^{k} v^{l} m^{i(j} m^{|k| l)}=$ $2\left(u^{i} m^{i j} v^{j}\right)^{2} \geq 0$. They constrain the signs of the elastic components in $\mathcal{M}^{i j k l}$ and also set upper and lower bounds on inelastic scattering amplitudes [21,22,29,43,44]. We will, however, show that these bounds are nonoptimal.

The goal of this Letter is to understand the exact boundary of $\mathbf{C}^{n^{4}}$, which is, in general, beyond superposed elastic bounds. In the presence of sufficient symmetries in the theory, an efficient way to do this is through the extremal positivity approach presented in Ref. [4], which determines the ERs of $\mathbf{C}^{n^{4}}$ using the symmetries of the EFT and constructs $\mathbf{C}^{n^{4}}$ from the ERs (see [51] for similar ideas). However, if operators that involve states not connected by any symmetries are considered, or if the theory possesses no symmetry at all, the number of ERs can become infinite, and this approach may not apply [22].
In this Letter, we propose a more general approach that does not rely on the symmetries of the theory and is thus immediately applicable to all multifield EFTs.

General bounds from spectrahedron.-Let us briefly outline this general approach. First, notice that, because cone $\mathbf{C}^{n^{4}}$ is convex, the dual cone of $\mathbf{C}^{n^{4}}$, defined as

$$
\mathbf{C}^{n^{4} *}=\left\{\mathcal{Q} \mid \mathcal{Q} \cdot \mathcal{M} \geq 0, \quad \forall \mathcal{M} \in \mathbf{C}^{n^{4}}\right\},
$$

is also convex and all bounds $\mathcal{Q} \cdot \mathcal{M} \geq 0$ for all $\mathcal{Q} \in \mathbf{C}^{n^{4} *}$ exactly describe the original cone $\mathbf{C}^{n^{4}}$. That is, the dual of dual cone $\mathbf{C}^{n^{4} *}$ equals the original cone $\mathbf{C}^{n^{4}}$. Therefore, instead of finding the $\mathbf{C}^{n^{4}}$ cone of amplitudes $\mathcal{M}$, we can equivalently work with the dual cone $\mathbf{C}^{n^{4} *}$. To determine salient cone $\mathbf{C}^{n^{4} *}$, we can simply find all its ERs, as positive linear combinations of these ERs generate the whole $\mathbf{C}^{n^{4} *}[52]$.

More precisely, since $\mathbf{C}^{n^{4}}$ is contained in the $\overrightarrow{\mathbf{S}}^{n^{4}}$ subspace, it is convenient to define the duality within $\overrightarrow{\mathbf{S}}^{n^{4}}$,

$$
\mathbf{Q}^{n^{4}} \equiv \mathbf{C}^{n^{4 *}}=\left\{\mathcal{Q} \in \overrightarrow{\mathbf{S}}^{n^{4}} \mid \mathcal{Q} \cdot \mathcal{M} \geq 0, \quad \forall \mathcal{M} \in \mathbf{C}^{n^{4}}\right\} .
$$

We now need to find $\mathbf{Q}^{n^{4}}$. For any $\mathcal{Q} \in \mathbf{Q}^{n^{4}}, \mathcal{Q} \cdot \mathcal{M} \geq 0 \Leftrightarrow$ $\mathcal{Q}^{i j k l} m^{i(j} m^{|k| l)} \geq 0 \Leftrightarrow 2 \mathcal{Q}^{i j k l} m^{i j} m^{k l} \geq 0$ for any $m$ (thanks to $\mathcal{Q} \in \overrightarrow{\mathbf{S}}^{n^{4}}$ ), which is equivalent to $\mathcal{Q} \succeq 0$. Therefore, we have $\mathbf{Q}^{n^{4}}=\mathbf{S}_{+}^{n^{2} \times n^{2}} \cap \overrightarrow{\mathbf{S}}^{n^{4}}$ which is known as a spectrahedron. Geometrically, a spectrahedron is the intersection of the cone of PSD matrices $\left(\mathbf{S}_{+}^{n^{2} \times n^{2}}\right)$ with an affine-linear space (in our case, $\overrightarrow{\mathbf{S}}^{n^{4}}$ ) and is a well-studied geometric object, intimately linked to semidefinite programming (SDP) - the latter is simply an optimization on a spectrahedron [53]. The complete and independent positivity bounds are simply $\mathcal{Q} \cdot \mathcal{M} \geq 0$ for all $\mathcal{Q} \in \operatorname{ext}\left(\mathbf{Q}^{n^{4}}\right)$.

We have essentially converted the problem of finding positivity bounds to a geometric problem: finding the ERs of a spectrahedron. Note that these ERs are in the dual space $\mathbf{Q}^{n^{4}}$ and are to be distinguished from the ERs of the physical amplitude space $\mathbf{C}^{n^{4}}$. The latter have been used in Ref. [4] to directly construct the boundary of $\mathbf{C}^{n^{4}}$. As we have mentioned, this procedure becomes cumbersome to use for theories with large $n$ but insufficient symmetries to determine the ERs. On the contrary, we will see that the new approach presented here does not have this limitation.

How do we search for the ERs in $\mathbf{Q}^{n^{4}}$ ? Just like a polyhedron, a spectrahedron has many (flat) faces of different dimensions. It has been shown in Ref. [55] that, for any point $\mathcal{Q}$ in a spectrahedron, there exists a unique face $F(\mathcal{Q})$ that contains $\mathcal{Q}$ with the lowest possible dimension and where $\operatorname{null}(\mathcal{Q})$ is constant [independent of where $\mathcal{Q}$ is on face $F(\mathcal{Q})$ ]. This provides a characterization of the faces and, in particular, the ERs (which are one-dimensional 
faces) of a spectrahedron. Let $u_{1}, u_{2}, \ldots, u_{k}$ be a basis of $\operatorname{null}(\mathcal{Q})$ and $\mathcal{Q}_{1}, \mathcal{Q}_{2}, \ldots, \mathcal{Q}_{m}$ be a basis of $\overrightarrow{\mathbf{S}}^{n^{4}}$, then the null space of the following $\left(n^{2} k\right) \times m$ matrix

$$
B=\left[\begin{array}{ccc}
\mathcal{Q}_{1} u_{1} & \cdots & \mathcal{Q}_{m} u_{1} \\
\vdots & \ddots & \vdots \\
\mathcal{Q}_{1} u_{k} & \cdots & \mathcal{Q}_{m} u_{k}
\end{array}\right]
$$

gives the linear subspace that contains $F(\mathcal{Q})$. If $\operatorname{null}(B)$ is one-dimensional, then $\mathcal{Q}$ is an ER. The positivity bounds are simply $\mathcal{Q} \cdot \mathcal{M} \geq 0$ for all such $\mathcal{Q}$ 's.

Toy model: Multiscalar.-Consider an EFT of $n$ scalar modes $\phi_{i=1, \ldots, n}$. At the tree level, the relevant operators are dim-8, and a basis can be chosen as $O_{i j k l}=$ $\partial_{\mu} \phi_{i} \partial^{\mu} \phi_{j} \partial_{\nu} \phi_{k} \partial^{\nu} \phi_{l}$, which has symmetry $O_{i j k l}=O_{j i k l}=$ $O_{i j l k}=O_{k l i j}$. Let us consider simply a $Z_{2}$ symmetric model $\left(\phi_{i} \rightarrow-\phi_{i}\right)$. The amplitude can be computed straightforwardly. We find $\mathcal{M}_{i i i i}=4 C_{i i i i}, \mathcal{M}_{i i j j}=\mathcal{M}_{i j j i}=\mathcal{M}_{j i i j}=$ $\mathcal{M}_{j j i i}=C_{i i j j}^{\prime} \equiv C_{i i j j}+\frac{1}{2} C_{i j i j}$, and $\mathcal{M}_{i j i j}=\mathcal{M}_{j i j i}=C_{i j i j}$. All other elements vanish.

The same $Z_{2}$ symmetry can be applied to its dual space, the spectrahedron $\mathbf{Q}^{n^{4}}$. For $n=2$, a general element in $\mathbf{Q}^{n^{4}}$ can be parametrized as

$$
\mathcal{Q}=\left[\begin{array}{llll}
x_{1} & x_{2} & & \\
x_{2} & x_{3} & \\
& & x_{4} & x_{2} \\
& & x_{2} & x_{4}
\end{array}\right], \quad x_{1,3} \geq 0, \quad x_{1} x_{3} \geq x_{2}^{2}, \quad x_{4} \geq\left|x_{2}\right|,
$$

where the rows (columns) correspond to the $i, j(k, l)$ pairs taking $(1,1),(2,2),(1,2),(2,1)$. The $2 \times 2$ block-diagonal structure is due to the $Z_{2}$ symmetry. Crossing symmetry is reflected the common matrix elements, while $\mathcal{Q} \succeq 0$ leads to the inequalities. Writing $\mathcal{Q} \equiv x_{i} \mathcal{Q}_{i}$, each $\mathcal{Q}$ can be represented by a $\vec{x}=\left(x_{1}, \ldots, x_{4}\right)$. From these inequalities, we can find the ERs: $\vec{x}_{e 1}(r)=\left(1, r, r^{2},|r|\right)$ and $\vec{x}_{e 2}=$ $(0,0,0,1)$, where $r$ is an arbitrary real number, and $\vec{x}_{e 1}(r)$ is extremal for any $r$. They are complete because any other $\vec{x}$ can be written as $\vec{x}=\left(x_{2}^{2} / x_{3}\right) \vec{x}_{e 1}\left(x_{3} / x_{2}\right)+$ $\left[x_{1}-\left(x_{2}^{2} / x_{3}\right)\right] \vec{x}_{e 1}(0)+\left(x_{4}-\left|x_{2}\right|\right) \vec{x}_{e 2}$, which is a positively weighted sum.

Each ER corresponds to an independent positivity bound. The second ER, $x_{e 2, i} \mathcal{Q}_{i} \cdot \mathcal{M} \geq 0$, simply gives $C_{1212}^{\prime} \geq 0$. The $r$-dependent ER $\vec{x}_{e 1}(r)$ gives $4 C_{2222} r^{2}+$ $4 C_{1122}^{\prime} r+2 C_{1212}|r|+4 C_{1111} \geq 0$. Together, they are equivalent to

$$
\begin{gathered}
C_{1111} \geq 0, \quad C_{2222} \geq 0, \quad C_{1212} \geq 0 \\
4 \sqrt{C_{1111} C_{2222}} \geq \pm\left(2 C_{1122}+C_{1212}\right)-C_{1212}
\end{gathered}
$$
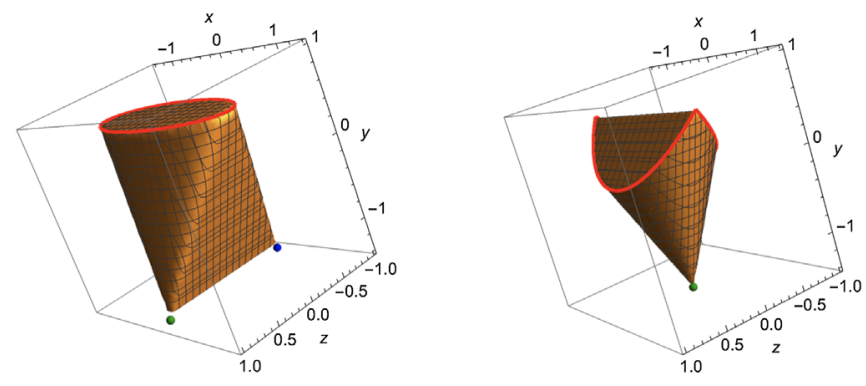

FIG. 1. Three-dimensional slice of $\mathbf{C}^{2^{4}}$ (left) and $\mathbf{Q}^{2^{4}}$ (right) for the biscalar toy example with $Z_{2}$ symmetry. The three axes in the left plot are taken to be $(x, y, z)=\left(2 \sqrt{6}\left(C_{1111}-C_{2222}\right)\right.$, $\left.\sqrt{2}\left(2 C_{1111}-C_{1212}+2 C_{2222}\right), \sqrt{3} C_{1122}^{\prime}\right)$, normalized to $4 C_{1111}+$ $C_{1212}+4 C_{2222}=1$. Those in the right plot are the same but with $C_{i j k l}^{(\prime)} \rightarrow Q_{i j k l}$.

As a quick application of this result, it improves the previous positivity bounds on the parameters of the Higgs-Dilaton inflationary model [50].

To illustrate the relation between $\mathbf{C}^{2^{4}}$ and its dual, in Fig. 1 we display the 3D cross sections of the physical amplitudes $\mathbf{C}^{2^{4}}$ and the spectrahedron $\mathbf{Q}^{2^{4}}$, which are both 4D cones. The two types of ERs of $\mathbf{Q}^{2^{4}}$ are highlighted by the red and green extreme points, respectively. The boundary of the $\mathbf{C}^{2^{4}}$ are dual to these ERs: a vertex in $\mathbf{Q}^{2^{4}}$ corresponds to a facet in $\mathbf{C}^{2^{4}}$ and vice versa, as implied by duality. Finding the full bounds is therefore equivalent to finding $\operatorname{ext}\left(\mathbf{Q}^{2^{4}}\right)$. On the other hand, the ERs of the physical amplitudes $\mathbf{C}^{2^{4}}$ are also highlighted. They can be of special physical interest, and we refer to Ref. [23] for potentially interesting phenomenological consequences. (More general cases with more modes and without $Z_{2}$ symmetry are presented in the Supplemental Material [56].)

Our approach always gives the complete bounds available from the dispersion relation. In contrast, the conventional positivity approach based on elastic scattering can be incomplete for a model with multiple modes. The elastic bounds are complete if and only if all elements of $\operatorname{ext}\left(\mathbf{Q}^{n^{4}}\right)$ can be written in form of $\mathcal{Q}_{u v}^{i j k l} \equiv u^{i} v^{j} u^{k} v^{l}+v^{i} u^{j} v^{k} u^{l}$. This can always be done for biscalar models, even without the $Z_{2}$ symmetry (see Supplemental Material [56]). However, this ceases to be true when there are three or more scalars. To see this, it suffices to give an example of $\mathcal{Q}$ being extremal in $\mathbf{Q}^{3^{4}}$ but not of the form of $\mathcal{Q}_{u v}$. One explicit example is $\mathcal{Q}_{\mathrm{ex}}=\sum_{\alpha=1}^{4} U_{\alpha}^{i j} U_{\alpha}^{k l}$, with the following four $U_{\alpha}$ matrices:

$\left[\begin{array}{lll}1 & 0 & 0 \\ 0 & 0 & 0 \\ 0 & 0 & 1\end{array}\right],\left[\begin{array}{lll}0 & 0 & 1 \\ 0 & 1 & 0 \\ 1 & 0 & 1\end{array}\right],\left[\begin{array}{lll}0 & 1 & 1 \\ 1 & 0 & 1 \\ 1 & 1 & 1\end{array}\right],\left[\begin{array}{ccc}0 & 1 & 1 \\ -1 & 0 & 0 \\ -1 & 0 & 0\end{array}\right]$.

$\mathcal{Q}_{\text {ex }}$ is a rank-4 matrix, so it cannot be written as some $\mathcal{Q}_{u v}$, which is at most rank-2 by definition. We will explain the 
physics interpretation of $\mathcal{Q}_{\mathrm{ex}}$ later, using the standard model (SM) flavor operators as an example.

We see that in the most general case, elastic positivity is incomplete for EFTs with more than two low-energy modes. In practice, however, the existence of symmetry relations can delay the appearance of nonelastic bounds. For example, the 4- $W$ operators presented in Ref. [4] contain nonelastic bounds. The $W$ boson carries two helicities and is charged under the adjoint of $\mathrm{SU}(2)$, which is equivalent to the fundamental of $\mathrm{SO}(3)$, thus the number of independent components in this case is six. However, if reducing the $\mathrm{SO}(3)$ to $\mathrm{SO}(2)$, which leads to four independent components left, there is no nonelastic bound any more.

General numerical method.-For a model with many low-energy modes, the optimal positivity bounds can be efficiently obtained numerically. To see this, note that $\mathcal{M}$ being in $\mathbf{C}^{n^{4}}$ is equivalent to $\mathcal{Q} \cdot \mathcal{M} \geq 0$ for all $\mathcal{Q} \in \mathbf{Q}^{n^{4}}$. This means we can get the optimal bounds by requiring the following semidefinite program:

$$
\begin{aligned}
\min & \mathcal{Q} \cdot \mathcal{M} \\
\text { subject to } & \mathcal{Q} \in \mathbf{Q}^{n^{4}}
\end{aligned}
$$

has a non-negative minimum. This solves the problem in polynomial time complexity and always gives the best bounds within given numerical accuracy, in contrast to the elastic positivity approach, which is NP-hard and leads to incomplete bounds.

It is sometimes useful to explicitly describe the boundary of $\mathbf{C}^{n^{4}}$. To this end, a Monte Carlo (MC) approach can be adopted in order to obtain a random sampling of linear bounds. To find an ER, one simply (1) picks a random point $\mathcal{Q}$ in $\mathbf{Q}^{n^{4}}$ and computes $F(\mathcal{Q})$ using Eq. (5).

(2) If $F(\mathcal{Q})$ is one-dimensional, then $\mathcal{Q}$ is on an ER; otherwise, take a random straight line in $F(\mathcal{Q})$ and find its intersection(s) with the boundary of $\mathbf{Q}^{n^{4}}$ (which is a SDP problem).

(3) Let $\mathcal{Q}$ be one of the intersection points and iterate, until an ER is found.

The iteration will take $\mathcal{Q}$ to a random ER. If the problem only has a finite number of bounds, this iteration will capture all bounds. This is often the case if one considers the self-interactions of some multiplet particle (see examples in Refs. [4,44]). For nonpolyhedral cones, we will get a sampling of bounds with a finite number of iterations.

Our new approach, in principle, captures all the information from the forward and twice-subtracted dispersion relation and improves many previous results based on elastic scattering. We now demonstrate this in subspaces of SMEFT.

SM gauge bosons.-In the SMEFT, positivity bounds at dim- 8 on gauge-boson operators are partially known $[4,20-22,29,44]$. To test our new approach, we consider parity-conserving four-gluon SMEFT operators. There are six relevant dim-8 operators (defined in Ref. [61]; see also Supplemental Material [56]), schematically of the form $\mathbf{G}^{4}$. The dim-6 operator $O_{G}=f^{A B C} G_{\mu}^{A \nu} G_{\nu}^{A \rho} G_{\rho}^{A \mu}$ can also contribute through diagrams with two insertions. The amplitude $\mathcal{M}$ can then be mapped to

$\vec{c} \equiv\left[\begin{array}{lllllll}C_{G^{4}}^{(1)} & C_{G^{4}}^{(2)} & C_{G^{4}}^{(3)} & C_{G^{4}}^{(4)} & C_{G^{4}}^{(7)} & C_{G^{4}}^{(8)} & c_{G}^{2}\end{array}\right]$,

where $C_{G^{4}}^{(i)}$ is the coefficient of $Q_{G^{4}}^{(i)}$ defined in Ref. [61], and $c_{G}$ is the coefficient of $O_{G}$.

Using the MC approach, we find 45 linear inequalities, which we have also verified with the symmetric extremal approach [4]. They can be written in the form of $\vec{x} \cdot \vec{c} \geq 0$, and the first six $\vec{x}$ vectors are

$$
\begin{array}{llc}
{[0,0,0,1,0,0,0]} & {[0,0,1,1,1,0,0]} & {[2,0,1,0,0,0,0]} \\
{[0,2,0,1,0,0,0]} & {[0,0,3,0,2,0,0]} & {[0,0,0,3,0,2,0]}
\end{array}
$$

and the other 39 are given in the Supplemental Material [56]. Previous results on parity-conserving operators based on selected elastic scattering in Ref. [29] can be reproduced already by the third to the sixth $\vec{x}$ vectors. We emphasize that this is a new result and an important step toward the full set of SMEFT positivity bounds.

The new approach is most powerful when multiple gauge-boson fields are incorporated, where the positivity cone is no longer polyhedral. A phenomenologically relevant case is the operators that characterize the anomalous quartic-gauge-boson couplings (aQGCs), which is an essential part of the electroweak program at the LHC (see Refs. [62-64] for recent results). Knowing positivity bounds for these operators will provide guidance for future experimental searches. For operators sourcing only the transversal modes, using the SDP approach, we find that the coefficient space is cut down to $0.681 \%$ of the total. This agrees with Ref. [22], where the same number is obtained by approximating the amplitude space by a polyhedral cone with a large number $\left[N \approx \mathcal{O}\left(10^{3}\right)\right]$ of edges and extrapolating $N \rightarrow \infty$, which is much less efficient. The full set of aQGC bounds can also be determined by the SDP approach. We will present it in a future work.

SM flavor sector.-A perhaps more relevant example is the SMEFT operators in the flavor sector. The SM fermions come with three generations, so full positivity bounds cannot be derived from elastic scattering of mixed flavors; flavor symmetry needs not be a symmetry of the SMEFT, so the symmetric extremal approach [4] does not apply. The SDP approach solves this problem. Consider one fermion species $f$, say the right-handed electron $f=e_{R}$, but for all three generations. Using the Fierz identity, the dim- 8 four-fermion operators can always be written as $O_{i j k l}=$ $\partial_{\mu}\left(\bar{f}_{i} \gamma_{\nu} f_{j}\right) \partial^{\mu}\left(\bar{f}_{k} \gamma^{\nu} f_{l}\right)$, where $i, j, k$, and $l$ are flavor 


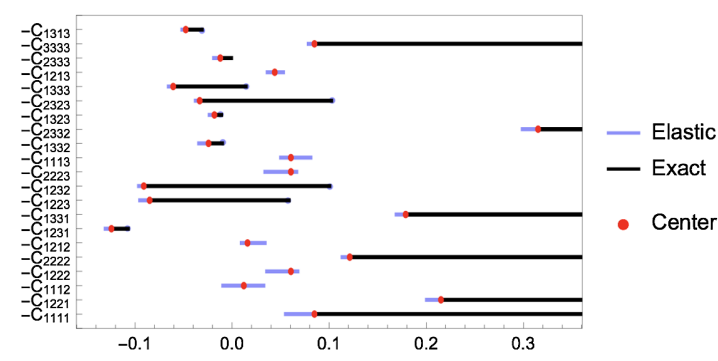

FIG. 2. Comparison of the elastic bounds ("Elastic") and the SDP bounds ("Exact"). The red dots ("Center") denote the set of coefficients $\vec{C}_{0}$, which saturates the nonelastic bound $\mathcal{Q}_{\text {ex }}$ given in Eq. (8).

indices. Since $O_{i j k l}=O_{k l i j}$, we only count the independent ones. The crossing symmetric amplitude $\mathcal{M}$ depends on 21 independent Wilson coefficients (see Supplemental Material [56]).

To illustrate the improvement of the new approach, we pick a set of coefficients $\vec{C}_{0}$ that saturates the nonelastic bound $\mathcal{Q}_{\text {ex }}$ given in Eq. (8) and display both elastic and the exact bounds in Fig. 2. These bounds are obtained by varying one operator at a time, while keeping the others fixed at $\vec{C}_{0}$, whose values are indicated with red dots. Elastic amplitudes are only bounded from below, while others are bounded from both sides. Since $\vec{C}_{0}$ is chosen to saturate the $\mathcal{Q}_{\mathrm{ex}}$ bound, the exact bounds could often uniquely fix the coefficients, so some exact bounds are not visible in the plot.

The new bound from $Q_{\mathrm{ex}}$ can be interpreted as coming from combining four channels between initial and final states $\left|I_{\alpha}\right\rangle=\left|F_{\alpha}\right\rangle=U_{\alpha}^{i j}|i\rangle \otimes|j\rangle$, for $\alpha=1,2,3,4$. The $U$ matrices are given in Eq. (8) and are at least rank 2, implying the two incoming particles are entangled. The $U_{1}$ matrix, for example, describes the scattering of the entangled state $\left|I_{1}\right\rangle=\left|F_{1}\right\rangle=|e\rangle \otimes|e\rangle+|\tau\rangle \otimes|\tau\rangle$. Individually, these states cannot be used to construct positivity bounds, because the $u$-channel contribution in the dispersion relation $U_{\alpha}^{i j} U_{\alpha}^{k l} m^{i l} m^{k j}$ is not positive semidefinite. However, the $\mathcal{Q}_{\text {ex }}$ tensor combines these channels together such that $\sum_{\alpha=1}^{4} U_{\alpha}^{i j} U_{\alpha}^{k l} \in \overrightarrow{\mathbf{S}}^{n^{4}}$ is crossing symmetric, which guarantees that both $s$ and $u$ channels are positive.

Positivity bounds for the flavor operators of the SMEFT are phenomenologically relevant, as the existence of flavorviolating effects (e.g., $\mu \rightarrow 3 e$ ) would set lower bounds on the flavor-conserving ones (e.g., $e^{+} e^{-} \rightarrow e^{+} e^{-}$), providing important guidance for future experiments [43]. While dim6 contributions potentially give the dominant contribution, future precision measurements are likely to have sufficient precision to simultaneously determine both dim- 6 and dim8 effects through global fits [23]. Novel observables have also been designed to extract dim- 8 information without being affected by the dim-6 ones [65]. Phenomenological studies for dim-8 SMEFT have started in the recent years $[23,33,35,47,65-71]$, and their interplay with positivity bounds may reveal crucial information about UV physics. Our new approach guarantees the best positivity bounds at dim- 8 and is thus crucial for fully capturing this information.

Summary.-We have shown that the full $s^{2}$ positivity bounds for EFTs with $n$ low-energy modes are given by the ERs of the spectrahedron $\mathbf{Q}^{n^{4}}$. We have formulated the problem of finding the optimal bounds as a semidefinite program, which can be efficiently solved in polynomial times. We have presented realistic examples and improved previous results in the areas of cosmology, LHC, and flavor physics (see the Supplemental Material [56] for more details, with Refs. [57-60] included there), which are all useful physical results by themselves. Our approach is straightforwardly applicable to all multifield EFTs and represents a crucial step toward fully extracting the positivity constraints for realistic EFTs with many degrees of freedom.

We would like to thank Anna Tokareva and Zi-Yue Wang for helpful discussions and comments. C. Z. is supported by IHEP under Contract No. Y7515540U1 and by National Natural Science Foundation of China (NSFC) under Grant No. 12035008. S. Y.Z. acknowledges support from the starting grants from University of Science and Technology of China under Grants No. KY2030000089 and No. GG2030040375, from the National Natural Science Foundation of China under Grants No. 11947301, No. 12075233, and No. 12047502, and from the Fundamental Research Funds for the Central Universities under Grant No. WK2030000036.

*lixu96@ihep.ac.cn

†haoxu@mail.ustc.edu.cn

*yangchengjie@ihep.ac.cn

scenzhang@ihep.ac.cn

zhoushy@ustc.edu.cn

[1] A. Adams, N. Arkani-Hamed, S. Dubovsky, A. Nicolis, and R. Rattazzi, Causality, analyticity and an IR obstruction to UV completion, J. High Energy Phys. 10 (2006) 014.

[2] T. N. Pham and T. N. Truong, Evaluation of the derivative quartic terms of the meson chiral lagrangian from forward dispersion relation, Phys. Rev. D 31, 3027 (1985).

[3] B. Ananthanarayan, D. Toublan, and G. Wanders, Consistency of the chiral pion pion scattering amplitudes with axiomatic constraints, Phys. Rev. D 51, 1093 (1995).

[4] C. Zhang and S.-Y. Zhou, Convex Geometry Perspective to the (Standard Model) Effective Field Theory Space, Phys. Rev. Lett. 125, 201601 (2020).

[5] C. de Rham, S. Melville, A. J. Tolley, and S.-Y. Zhou, Positivity bounds for scalar field theories, Phys. Rev. D 96, 081702(R) (2017).

[6] C. de Rham, S. Melville, A. J. Tolley, and S.-Y. Zhou, UV complete me: Positivity bounds for particles with spin, J. High Energy Phys. 03 (2018) 011. 
[7] A. J. Tolley, Z.-Y. Wang, and S.-Y. Zhou, New positivity bounds from full crossing symmetry, J. High Energy Phys. 05 (2021) 255.

[8] S. Caron-Huot and V. Van Duong, Extremal effective field theories, J. High Energy Phys. 05 (2021) 280.

[9] N. Arkani-Hamed, T.-C. Huang, and Y.-t. Huang, The EFThedron, J. High Energy Phys. 05 (2021) 259.

[10] B. Bellazzini, J. Elias Miró, R. Rattazzi, M. Riembau, and F. Riva, Positive moments for scattering amplitudes, Phys. Rev. D 104, 036006 (2021).

[11] L. Vecchi, Causal versus analytic constraints on anomalous quartic gauge couplings, J. High Energy Phys. 11 (2007) 054.

[12] A. V. Manohar and V. Mateu, Dispersion relation bounds for pi pi scattering, Phys. Rev. D 77, 094019 (2008).

[13] A. Nicolis, R. Rattazzi, and E. Trincherini, Energy's and amplitudes' positivity, J. High Energy Phys. 05 (2010) 095; 11 (2011) 128(E).

[14] B. Bellazzini, C. Cheung, and G. N. Remmen, Quantum gravity constraints from unitarity and analyticity, Phys. Rev. D 93, 064076 (2016).

[15] B. Bellazzini, Softness and amplitudes positivity for spinning particles, J. High Energy Phys. 02 (2017) 034.

[16] C. Cheung and G. N. Remmen, Positivity of CurvatureSquared Corrections in Gravity, Phys. Rev. Lett. 118, 051601 (2017).

[17] B. Bellazzini, M. Lewandowski, and J. Serra, Amplitudes' Positivity, Weak Gravity Conjecture, and Modified Gravity, Phys. Rev. Lett. 123, 251103 (2019).

[18] L. Alberte, C. de Rham, S. Jaitly, and A. J. Tolley, Positivity bounds and the massless spin-2 pole, Phys. Rev. D 102, 125023 (2020).

[19] A. Hebbar, D. Karateev, and J. Penedones, Spinning $S$-matrix bootstrap in 4d, arXiv:2011.11708.

[20] C. Zhang and S.-Y. Zhou, Positivity bounds on vector boson scattering at the LHC, Phys. Rev. D 100, 095003 (2019).

[21] Q. Bi, C. Zhang, and S.-Y. Zhou, Positivity constraints on aQGC: Carving out the physical parameter space, J. High Energy Phys. 06 (2019) 137.

[22] K. Yamashita, C. Zhang, and S.-Y. Zhou, Elastic positivity vs extremal positivity bounds in SMEFT: A case study in transversal electroweak gauge-boson scatterings, J. High Energy Phys. 01 (2021) 095.

[23] B. Fuks, Y. Liu, C. Zhang, and S.-Y. Zhou, Positivity in electron-positron scattering: Testing the axiomatic quantum field theory principles and probing the existence of UV states, Chin. Phys. C 45, 023108 (2021).

[24] C. de Rham, S. Melville, A. J. Tolley, and S.-Y. Zhou, Massive Galileon positivity bounds, J. High Energy Phys. 09 (2017) 072.

[25] C. de Rham, S. Melville, A. J. Tolley, and S.-Y. Zhou, Positivity bounds for massive spin-1 and spin-2 fields, J. High Energy Phys. 03 (2019) 182.

[26] Y.-J. Wang, F.-K. Guo, C. Zhang, and S.-Y. Zhou, Generalized positivity bounds on chiral perturbation theory, J. High Energy Phys. 07 (2020) 214.

[27] Z.-Y. Wang, C. Zhang, and S.-Y. Zhou, Generalized elastic positivity bounds on interacting massive spin-2 theories, J. High Energy Phys. 04 (2021) 217.
[28] J. Distler, B. Grinstein, R. A. Porto, and I. Z. Rothstein, Falsifying Models of New Physics via WW Scattering, Phys. Rev. Lett. 98, 041601 (2007).

[29] G. N. Remmen and N. L. Rodd, Consistency of the standard model effective field theory, J. High Energy Phys. 12 (2019) 032.

[30] C. Cheung and G. N. Remmen, Positive signs in massive gravity, J. High Energy Phys. 04 (2016) 002.

[31] B. Bellazzini, F. Riva, J. Serra, and F. Sgarlata, Beyond Positivity Bounds and the Fate of Massive Gravity, Phys. Rev. Lett. 120, 161101 (2018).

[32] J. Bonifacio, K. Hinterbichler, and R. A. Rosen, Positivity constraints for pseudolinear massive spin-2 and vector Galileons, Phys. Rev. D 94, 104001 (2016).

[33] B. Bellazzini, F. Riva, J. Serra, and F. Sgarlata, The other effective fermion compositeness, J. High Energy Phys. 11 (2017) 020.

[34] J. Bonifacio and K. Hinterbichler, Bounds on amplitudes in effective theories with massive spinning particles, Phys. Rev. D 98, 045003 (2018).

[35] B. Bellazzini and F. Riva, $Z Z$ and $Z \gamma$ still haven't found what they are looking for, Phys. Rev. D 98, 095021 (2018).

[36] S. Melville and J. Noller, Positivity in the sky, Phys. Rev. D 101, 021502 (2020).

[37] C. de Rham and A. J. Tolley, Speed of gravity, Phys. Rev. D 101, 063518 (2020).

[38] L. Alberte, C. de Rham, A. Momeni, J. Rumbutis, and A. J. Tolley, Positivity constraints on interacting spin-2 fields, J. High Energy Phys. 03 (2020) 097.

[39] L. Alberte, C. de Rham, A. Momeni, J. Rumbutis, and A. J. Tolley, Positivity constraints on interacting pseudo-linear spin-2 fields, J. High Energy Phys. 07 (2020) 121.

[40] W.-M. Chen, Y.-T. Huang, T. Noumi, and C. Wen, Unitarity bounds on charged/neutral state mass ratios, Phys. Rev. D 100, 025016 (2019).

[41] Y.-t. Huang, J.-Y. Liu, L. Rodina, and Y. Wang, Carving out the space of open-string $S$ matrix, J. High Energy Phys. 04 (2021) 195.

[42] J. Tokuda, K. Aoki, and S. Hirano, Gravitational positivity bounds, J. High Energy Phys. 11 (2020) 054.

[43] G. N. Remmen and N. L. Rodd, Flavor Constraints from Unitarity and Analyticity, Phys. Rev. Lett. 125, 081601 (2020).

[44] T. Trott, Causality, unitarity and symmetry in effective field theory, arXiv:2011.10058.

[45] A. Guerrieri, J. Penedones, and P. Vieira, S-matrix bootstrap for effective field theories: Massless pions, J. High Energy Phys. 06 (2021) 088.

[46] J. Gu and L.-T. Wang, Sum rules in the standard model effective field theory from helicity amplitudes, J. High Energy Phys. 03 (2021) 149.

[47] J. Gu, L.-T. Wang, and C. Zhang, An unambiguous test of positivity at lepton colliders, arXiv:2011.03055.

[48] Q. Bonnefoy, E. Gendy, and C. Grojean, Positivity bounds on minimal flavor violation, J. High Energy Phys. 04 (2021) 115.

[49] M. Herrero-Valea, R. Santos-Garcia, and A. Tokareva, Massless positivity in graviton exchange, arXiv:2011.11652.

[50] M. Herrero-Valea, I. Timiryasov, and A. Tokareva, To positivity and beyond, where Higgs-Dilaton inflation has 
never gone before, J. Cosmol. Astropart. Phys. 11 (2019) 042.

[51] B. Bellazzini, L. Martucci, and R. Torre, Symmetries, sum rules and constraints on effective field theories, J. High Energy Phys. 09 (2014) 100.

[52] M. Krein and D. Milman, On extreme points of regular convex sets, Stud. Math. 9, 133 (1940).

[53] For applications of SDP in conformal bootstrapping, see [54] and references therein.

[54] D. Poland, S. Rychkov, and A. Vichi, The conformal bootstrap: Theory, numerical techniques, and applications, Rev. Mod. Phys. 91, 015002 (2019).

[55] M. Ramana and A. J. Goldman, Some geometric results in semidefinite programming, J. Global Optim. 7, 33 (1995).

[56] See Supplemental Material at http://link.aps.org/ supplemental/10.1103/PhysRevLett.127.121601 for more details about general multi-scalar EFTs, SM gauge bosons, SM fermions and applications in constraining spin-2 EFTs, which includes Refs. [57-60].

[57] L. Alberte, C. de Rham, A. Momeni, J. Rumbutis, and A. J. Tolley, EFT of interacting spin-2 fields, J. High Energy Phys. 01 (2020) 131.

[58] C. de Rham, G. Gabadadze, and A. J. Tolley, Resummation of Massive Gravity, Phys. Rev. Lett. 106, 231101 (2011).

[59] D. Hilbert, Ueber die darstellung definiter formen als summe von formenquadraten, Math. Ann. 32, 342 (1888).

[60] L. Keltner and A. J. Tolley, UV properties of Galileons: Spectral densities, arXiv:1502.05706.

[61] C. W. Murphy, Dimension-8 operators in the standard model effective field theory, J. High Energy Phys. 10 (2020) 174.

[62] A. M. Sirunyan et al. (CMS Collaboration), Search for anomalous electroweak production of vector boson pairs in association with two jets in proton-proton collisions at 13 TeV, Phys. Lett. B 798, 134985 (2019).

[63] CMS Collaboration, Measurements of production cross sections of same-sign $W W$ and $W Z$ boson pairs in association with two jets in proton-proton collisions at $\sqrt{s}=13$ TeV, Phys. Lett. B 809, 135710 (2020).

[64] A. M. Sirunyan et al. (CMS Collaboration), Measurement of the cross section for electroweak production of a $Z$ boson, a photon and two jets in proton-proton collisions at $\sqrt{\mathrm{s}}=$ $13 \mathrm{TeV}$ and constraints on anomalous quartic couplings, J. High Energy Phys. 06 (2020) 076.

[65] S. Alioli, R. Boughezal, E. Mereghetti, and F. Petriello, Novel angular dependence in Drell-Yan lepton production via dimension-8 operators, Phys. Lett. B 809, 135703 (2020).

[66] J. Ellis, N. E. Mavromatos, and T. You, Light-by-Light Scattering Constraint on Born-Infeld Theory, Phys. Rev. Lett. 118, 261802 (2017).

[67] J. Ellis and S.-F. Ge, Constraining Gluonic Quartic Gauge Coupling Operators with $g g \rightarrow \gamma \gamma$, Phys. Rev. Lett. 121, 041801 (2018).

[68] J. Ellis, S.-F. Ge, H.-J. He, and R.-Q. Xiao, Probing the scale of new physics in the $Z Z \gamma$ coupling at $e^{+} e^{-}$colliders, Chin. Phys. C 44, 063106 (2020).

[69] J. Ellis, H.-J. He, and R.-Q. Xiao, Probing new physics in dimension-8 neutral gauge couplings at $e^{+} e^{-}$colliders, Sci. China Phys. Mech. Astron. 64, 221062 (2021).

[70] T. Corbett, A. Helset, A. Martin, and M. Trott, EWPD in the SMEFT to dimension eight, J. High Energy Phys. 06 (2021) 076.

[71] C. Hays, A. Martin, V. Sanz, and J. Setford, On the impact of dimension-eight SMEFT operators on Higgs measurements, J. High Energy Phys. 02 (2019) 123. 